\title{
Wissenschaftliche App-Generatoren: Eine Option für die Wirtschaft?
}

Mit App-Generatoren lassen sich Apps ohne oder nur mit geringen Programmierkenntnissen erstellen. Gerade für kleine und mittlere Unternehmen (KMUs) und Non-Profit-Organisationen (NGOs) können diese Werkzeuge eine vielversprechende Alternative zur kostspieligen Fremdentwicklung sein. Neben einer Vielzahl kommerzieller Anbieter haben, der breiten Öffentlichkeit bislang weitgehend verborgen, auch zahlreiche Wissenschaftler App-Generatoren entwickelt. Auf Basis einer aktuellen, umfassenden Literaturrecherche gibt dieser Beitrag einen Überblick über die aus der Wissenschaft stammenden App-Generatoren und untersucht diese auf ihre Praxistauglichkeit.

\section{Tobias Friedl und Sebastian Floerecke}


Für die klassische Erstellung einer App sind fundierte Programmierkenntnisse erforderlich. Nachdem darüber verhältnismäßig wenige Personen verfügen, muss häufig der Weg einer kostspieligen Fremdentwicklung durch externe Dienstleister gewählt werden. Eine vielversprechende, deutlich kostengünstigere Alternative gerade für KMUs und NGOs wie Schulen, Museen und Vereine ist die Eigenerstellung mithilfe von App-Generatoren.

\section{Was sind App-Generatoren?}

App-Generatoren sind Anwendungen, mit denen Apps per grafischer Oberfläche erstellt werden können. Kenntnisse über betriebssystemspezifische Programmierschnittstellen und die Syntax von Programmiersprachen sind nicht nötig. Die Modellierung von Kontrollstrukturen wie Verzweigungen und Schleifen entfällt oder ist auf eine ganz einfache Art möglich. App-Generatoren bieten somit ausschließlich vordefinierte Module, die über Parameter angepasst werden können.

$\mathrm{Zu}$ den verwendbaren Modulen beziehungsweise Funktionalitäten zählen insbesondere das Definieren von DropdownMenüs, das Einbinden sowohl statischer Inhalte wie Hintergrund- und Galeriebilder, Preislisten und Öffnungszeiten als auch dynamischer Inhalte wie Navigationskarten, RSS-Feeds und sozialen Netzwerken sowie das Integrieren von PushNachrichten und Coupon-Systemen.

\section{Umkämpfter App-Generatorenmarkt}

Einer kürzlich von Friedl und Floerecke [1] veröffentlichten Marktstudie zufolge befinden sich derzeit knapp 70 Anbieter auf dem globalen App-Generatorenmarkt. Wie die über die vergangenen Jahre hinweg spürbare Marktbereinigung zeigt, handelt es sich hierbei um einen dynamischen und hart umkämpften Markt. Insgesamt wurden ganze $11 \%$ aller kostenlosen Android-Apps mit kommerziellen App-Generatoren erstellt [2]. Was der Öffentlichkeit bislang weitgehend verborgen blieb: Auch Wissenschaftler haben eigene App-Generatoren entwickelt. Ungeklärt ist dabei die Frage, inwieweit sich diese für die betriebliche Praxis eignen.

\section{Kernthese 1}

Gerade für KMUs und NGOs sind App-Generatoren eine vielversprechende Alternative zur kostspieligen Fremdentwicklung.

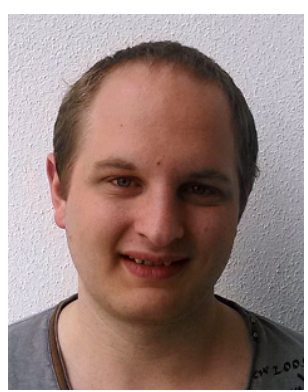

Tobias Friedl M.Sc. ${ }^{1}$

ist Programmierer und Customizer am Zentrum für Informationstechnologie und Medienmanagement der Universität Passau. Aktuell beschäftigt er sich mit der Einführung eines neuen CampusManagement-Systems. Davor forschte er als wissenschaftlicher Mitarbeiter am Lehrstuhl für Wirtschaftsinformatik der Universität Passau auf dem Gebiet der App-Entwicklung. tobias.friedl@uni-passau.de

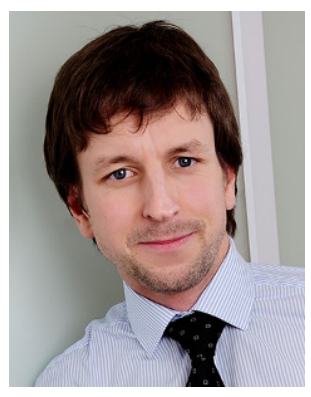

Dr. Sebastian Floerecke ${ }^{1}(\square)$ arbeitet bei der Flughafen München $\mathrm{GmbH}$ als IT Solution Manager und ist Autor zahlreicher Veröffentlichungen $z u$ den Themengebieten Cloud-Computing, hybride Produkte, Apps, Anforderungsmanagement sowie Business-Ökosysteme und Geschäftsmodelle. Zuvor war er als wissenschaftlicher Mitarbeiter und Doktorand am Lehrstuhl für Wirtschaftsinformatik der Universität Passau in Forschung und Lehre tätig. sebastian.floerecke@uni-passau.de

${ }^{1}$ Universität Passau, 94032 Passau, Deutschland 


\section{Kernthese 2}

In der Spitzenforschung wird App-Generatoren nur eine geringe Bedeutung beigemessen.

\section{Kein Thema für die Spitzenforschung}

Im Rahmen der umfassenden Literaturrecherche im Februar und März dieses Jahres wurden 62 App-Generatoren, ähnlich der Größe des Marktangebots, identifiziert. Diese wurden in 183 wissenschaftlichen Publikationen thematisiert. Gesucht wurde in den führenden wissenschaftlichen Datenbanken ACM Digital Library, IEEE Explorer, SpringerLink, ScienceDirect und Google Scholar ab dem Jahr 2007 - Erscheinungsjahr des ersten iPhones und damit Beginn des App-Booms.

Was sich dabei übergeordnet herausstellte: Die Forschungsbeiträge wurden fast ausschließlich bei niedrig eingestuften Journalen oder Konferenzen veröffentlicht. In der Spitzenforschung wird diesem Thema offensichtlich keine große Bedeutung beigemessen. In den allermeisten Fällen wurden die App-Generatoren innerhalb von Dissertationsprojekten (zum Beispiel MobiMed [3] oder Mobia Modeler [4]) entwickelt. Aber auch Master- (zum Beispiel DETACH [5]) und Bachelorarbeiten (zum Beispiel Mobile Mashup Editor [6]) waren darunter. Teils entstanden die App-Generatoren im Rahmen staatlich geförderter Forschungsprojekte. Kooperationen mit Industriepartnern waren und sind bis heute selten. Wenn es Kooperationen gab, dann vor allem mit einzelnen Museen, Schulen oder kleineren medizinischen Einrichtungen.

\section{Technische Eigenschaften der wissen- schaftlichen App-Generatoren und der erzeugbaren Apps}

- App-Arten: Rund $27 \%$ der App-Generatoren produzieren Web-Apps, 21 \% hybride Apps und $61 \%$ native Apps. Bei den kommerziellen App-Generatoren dominieren dagegen die hybriden Apps. Native Funktionen zum Zugriff auf die Gerätehardware, kombiniert mit Inhalten in HTML und CSS (hybride Apps), sind für kommerzielle App-Generatoranbieter offenbar die einfachste Möglichkeit zur Generierung von Apps für mehrere Betriebssysteme.

- Betriebssysteme: Mit etwa 21 \% der App-Generatoren lassen sich Apps für iOS und mit rund 47 \% für Android erstellen. Manche Werkzeuge können auch mehrere Betriebssysteme gleichzeitig bedienen. Wenig überraschend befinden sich im Gegensatz zum Praxisangebot einige App-Generatoren darunter, die auch oder ausschließlich nicht mehr gewartete mobile Plattformen wie Windows Mobile 5, Windows CE oder BlackBerry OS adressieren.

- Anwendungsbereich: Die Mehrheit der App-Generatoren erlaubt die Erstellung von Apps nur für einen speziellen Anwendungsbereich - Automatisierungsaufgaben (16\%), formularbasierte Datenerhebung (29\%) sowie Verknüpfung von Inhalten aus verschiedenen Internetquellen via Mashups (23\%). Auch wenn die restlichen Werkzeuge (32\%) keinen speziellen Anwendungsbereich aufweisen, kann von einer universellen Verwendbarkeit der damit geschaffenen Apps schon allein wegen der beschränkten Menge an Features beziehungsweise deren hochgradige Spezialisierung jedoch nicht die Rede sein.

- Hauptzielgruppen: Die Hauptzielgruppen der Forscher sind NGOs wie Schulen und Museen sowie Privatpersonen, die Mashups erstellen oder mit dem Mobilgerät insbesondere medizinische Daten erheben möchten (wie das Angebot an Apps im medizinischen Bereich speziell für Senioren ist, untersuchten die Autoren in [7]). Auch der Internet-ofThings(IoT)-Bereich wird des Öfteren angesprochen.

- Entwicklungsplattform: Bei mehr als der Hälfte (56\%) der App-Generatoren wird in Cloud-Anwendungen im Browser entwickelt. Daneben existieren Desktop-Anwendungen (26\%). In $21 \%$ der Fälle dient das Mobilgerät nicht nur als Ziel-, sondern auch als Entwicklungsplattform. Unter den kommerziellen App-Generatoren befinden sich dagegen beinahe ausschließlich Cloud-Anwendungen.

\section{Ernüchterndes Ergebnis}

Die Antwort auf die Frage, inwieweit sich die aus der Wissenschaft stammenden App-Generatoren für Praktiker eignen, fällt ernüchternd aus: überhaupt nicht. Die Gründe dafür sind vielfältig.

\section{Handlungsempfehlungen}

- Für einfach gehaltene Apps mit statischen und wenig komplexen, dynamischen Inhalten sind kommerzielle App-Generatoren bestens geeignet.

- Die Wahl eines kommerziellen App-Generatoranbieters muss mit äußerster Sorgfalt unter Einbeziehung nicht zu unterschätzender Risiken erfolgen.

- Forscher sollten sich verstärkt darauf konzentrieren, was Praktiker tatsächlich benötigen, und nicht darauf, was theoretisch möglich wäre. 
Ein grundsätzliches Problem stellt die fehlende öffentliche Verfügbarkeit dar. Von den 62 identifizierten App-Generatoren wurden lediglich sieben überhaupt zur öffentlichen Nutzung freigegeben. Davon sind aktuell nur mehr zwei aktiv. Aber auch wenn sämtliche App-Generatoren zur Verfügung gestellt worden wären, würde sich die Situation heute nicht viel anders darstellen. Denn die App-Generatoren entstanden im Rahmen von Forschungsprojekten von Universitätslehrstühlen und studentischen Abschlussarbeiten. Mit deren Abschluss endete auch jeweils die Weiterentwicklung und die Wartung des geschaffenen App-Generators. Die jährlich neu erscheinenden Betriebssystemversionen von insbesondere Android und iOS und die sich häufig ändernden Richtlinien der App-Stores zur Veröffentlichung von Apps machen jedoch regelmäßige Anpassungen an Apps und folglich ebenfalls an den App-Generatoren zwingend erforderlich. Aber auch während der Projektlaufzeit kann seitens der Forscher allein schon aufgrund mangelnder Ressourcen kein für Praktiker allerdings unabdingbarer Support geleistet werden.

Darüber hinaus ist die Menge an Features und die User Experience im Vergleich zu den kommerziellen Angeboten begrenzt. Ein wesentlicher Grund: Bei den meisten Werkzeugen handelt es sich lediglich um Prototypen, die zur Evaluierung von Konzepten, wie etwa das Zusammenfügen von als Puzzleteile repräsentierten Funktionsbausteinen, geschaffen wurden. Oder schlichtweg um zu zeigen, wie gut sich eine domänenspezifische Sprache (eine formale, für eine bestimmte Domäne erstellte und meist leicht lesbare Sprache, der eine Eignung für die Endbenutzerentwicklung attestiert wird) für einen speziellen Fachbereich (zum Beispiel bei MobiCloud [8]) oder eine Modellierungssprache (zum Beispiel bei MAML [9]) grundsätzlich für die Endbenutzerentwicklung eignet. Von diesen Prototypen wurden allerdings keine oder nur oberflächliche Evaluationen durchgeführt. Die teils über deren Qualität und Nutzbarkeit in den entsprechenden wissenschaftlichen Publikationen getätigten Aussagen sind daher nur wenig belastbar. Ein weiterer Grund für die geringe Menge der Features ist die Tatsache, dass die App-Generatoren häufig auf einen ganz spezifischen Anwendungsbereich wie etwa Museumsführungen (zum Beispiel Pud Guide [10] und Cicero Designer [11]) oder einfache Automatisierungen (zum Beispiel IVO [12] und MicroApp [13]), vielfach im IoTBereich, abzielen.

Ein weiteres Hindernis für die Wirtschaft besteht darin, dass gut die Hälfte der untersuchten App-Generatoren Apps ausliefert, die nicht eigenständig lauffähig sind. Stattdessen
Die Hauptgründe für die unzulängliche Praxistauglichkeit der wissenschaftlichen App-Generatoren auf einen Blick

- prototypischer Charakter

- fehlende öffentliche Verfügbarkeit

- fehlender Support

- geringe Menge an Features

- unzureichende User Experience

- Beschränkung auf einen spezifischen Anwendungsbereich

- oftmals umständliche Inbetriebnahme („Player-App“ notwendig)

- Einstellung der Weiterentwicklung und der Wartung nach Ende des jeweiligen Forschungsprojekts

- häufig veraltete Zielbetriebssysteme

muss der Anwender nicht gerade nutzerfreundlich zusätzlich eine sogenannte „Player-App“ - je nach Implementierung ein Interpreter oder eine Laufzeitumgebung - installieren und ausführen.

\section{Bedeutung der Untersuchungsergebnisse für Wirtschaft und Wissenschaft}

Damit KMUs, NGOs und sonstige Akteure aus der Wirtschaft die von der Wissenschaft entwickelten App-Generatoren überhaupt einsetzen könnten, müssen die Universitäten und Hochschulen diese zunächst als kommerzielle oder Open-Source-Versionen verfügbar machen. Dazu müssen sie zwangsläufig Forschungskooperationen mit Unternehmen eingehen. Der Wirtschaft hilft es wenig, wenn lediglich kleine Insellösungen konzipiert, umgesetzt und nach Projektende nicht weiterverfolgt werden. Die Wissenschaft sollte sich deshalb zukünftig verstärkt darauf konzentrieren, was Praktiker für ihre Zwecke tatsächlich benötigen und nicht was theoretisch machbar wäre. Dieser Umstand manifestiert sich darin, dass bislang nur eine geringe Menge der wissenschaftlichen App-Generatoren hinsichtlich ihres Praxisnutzens untersucht

\section{Kernthese 3}

Der Wirtschaft hilft es wenig, wenn lediglich kleine Insellösungen konzipiert, umgesetzt und nach Projektende nicht weiterverfolgt werden. 


\section{Zusammenfassung}

- App-Generatoren sind Anwendungen, mit denen Apps ohne Programmierkenntnisse per grafischer Oberfläche erstellt werden können.

- Neben einer Vielzahl kommerzieller Anbieter haben, der Öffentlichkeit bislang weitgehend verborgen, auch zahlreiche Wissenschaftler App-Generatoren entwickelt.

- Nachdem die Wissenschaft so gut wie keine Hilfestellung und Werkzeuge für die betriebliche Praxis liefert, müssen sich Praktiker mit dem durchaus beträchtlichen kommerziellen Angebot begnügen.

wurde. Da überrascht es nicht, dass das Interesse der Forschung am kommerziellen Werkzeugangebot gering ist: Nur in Publikationen zu zehn der 62 App-Generatoren ist überhaupt von Werkzeugen aus der Praxis die Rede.

Damit bleibt Praktikern zumindest mittelfristig nur die Möglichkeit, einen App-Generator aus der Wirtschaft zu verwenden. Die gute Nachricht ist, dass sowohl der nationale als auch der internationale Markt ein beträchtliches, wenn auch etwas unübersichtliches Angebot bereithält. Die Auswahl eines geeigneten App-Generators ist aufgrund deren unterschiedlichen Schwerpunkte und der heterogenen Anforderungen von KMUs und NGOs allerdings mit etwas Zeitaufwand verbunden. Führende App-Generatoren sind Andromo und Seattle Clouds. Der Schweizer Agentur AppBrain [14] zufolge, Herausgeber zahlreicher Studien rund um den App-Markt, wurden damit 1,3 \% beziehungsweise 0,9 \% aller kostenlosen Android-Apps erzeugt.

Kommerzielle App-Generatoren bieten grundsätzlich vorgegebene parametrisierbare Module. Damit lassen sich natürlich keine komplexen, hochgradig individualisierten Apps erstellen. Doch für eher einfach gehaltene Apps mit statischen Inhalten, Kontaktformular und Navigationskarte reichen sie allemal. Allerdings sind mit der Verwendung von App-Generatoren auch gewisse Risiken verbunden [1]: Dazu zählen Datenschutz- und Datensicherheitsrisiken, die fehlende Umzugsmöglichkeit der App zwischen Anbietern beziehungsweise Erwerbbarkeit des Quellcodes bei Einstellung des App-Generators, gegebenenfalls versteckte Kosten und die Aspekte Verschlüsselung, Berechtigungen und Nutzer-Tracking.

Auch wenn dieser Untersuchung zufolge das Angebot wissenschaftlicher App-Generatoren für die Wirtschaft aus den verschiedensten Gründen ungeeignet ist, können Praktiker auf eine Vielzahl kommerzieller App-Generatoren zurückgreifen. Die Anbieterauswahl sollte mit Sorgfalt erfolgen die Risiken müssen hinreichend berücksichtigt und behandelt werden. Dann steht KMUs und NGOs der Eigenerstellung einer App ohne Programmierkenntnisse und damit ohne Inanspruchnahme teurer externer Entwickler nichts mehr im Wege.

Funding. Open Access funding enabled and organized by Projekt DEAL.

Open Access. Dieser Artikel wird unter der Creative Commons Namensnennung 4.0 International Lizenz veröffentlicht, welche die Nutzung, Vervielfältigung, Bearbeitung, Verbreitung und Wiedergabe in jeglichem Medium und Format erlaubt, sofern Sie den/die ursprünglichen Autor(en) und die Quelle ordnungsgemäß nennen, einen Link zur Creative Commons Lizenz beifügen und angeben, ob Änderungen vorgenommen wurden.

Die in diesem Artikel enthaltenen Bilder und sonstiges Drittmaterial unterliegen ebenfalls der genannten Creative Commons Lizenz, sofern sich aus der Abbildungslegende nichts anderes ergibt. Sofern das betreffende Material nicht unter der genannten Creative Commons Lizenz steht und die betreffende Handlung nicht nach gesetzlichen Vorschriften erlaubt ist, ist für die oben aufgeführten Weiterverwendungen des Materials die Einwilligung des jeweiligen Rechteinhabers einzuholen.

Weitere Details zur Lizenz entnehmen Sie bitte der Lizenzinformation auf http://creativecommons.org/licenses/by/4.0/ deed.de.

\section{Literatur}

[1] Friedl, T., \& Floerecke, S. (2020). Endbenutzerwerkzeuge zur App-Entwicklung: Grundlagen, Kategorisierung und Risiken. OBJEKTspektrum, 3, 34-40.

[2] Oltrogge, M., Derr, E., Stransky, C., Acar, Y., Fahl, S., Rossow, C., Pellegrino, G., Bugiel, S., \& Backes, M. (2018). The rise of the citizen developer: assessing the security impact of online app generators. 39. IEEE Symposium on Security and Privacy, San Francisco.

[3] Hernadez, F. (2013). MobiMed: Framework for rapid application development of medical mobile apps. Dissertation, Internationale Universität Florida, USA.

[4] Balagtas-Fernandez, F. T., \& Hussmann, H. (2008). Model-driven development of mobile applications. 23. IEEE/ACM International Conference on Automated Software Engineering, L'Aquila. 
[5] Fernandes, F. (2013). Detach: design tool for smartphone application composition. Masterarbeit, Universität Lissabon, Portugal.

[6] Kaltofen, S. (2010). Design and implementation of an end-user programming software system to create and deploy cross-platform mobile mashups. Bachelorarbeit, Linné-Universität, Schweden.

[7] Friedl, T., Floerecke, S., \& Lehner, F. (2015). Mobile Anwendungen zur Gesundheitsförderung von Senioren - Eine Untersuchung des aktuellen App-Angebots. eHealth Mobility Journal, 3, 128-131.

[8] Manjunatha, A., Ranabahu, A., Sheth, A., \& Thirunarayan, K. (2010). A domain specific language based method to develop cloudmobile hybrid applications. Write State Universität, USA.

[9] Rieger, C., \& Kuchen, H. (2018). A process-oriented modeling approach for graphical development of mobile business apps. Computer Languages, Systems \& Structures, 2018(53), 43-58.

[10] Celentano, A., Orsini, R., Pittarello, F., \& Barbieri, G. (2009). Design and evaluation of a mobile art guide on ipod touch. Interaction Design and Architecture(s) Journal, 5-6, 77-80.

[11] Ghiani, G., Paternò, F., Santoro, C., \& Spano, L. D. (2009). biCicero: a location-aware, multi-device museum guide. Interacting with Computers, 21(4), 288-303.

[12] Realinho, V., Romão, T., Birra, F., \& Dias, A. E. (2011). Rapid development of mobile context-aware applications with IVO. 8. International Conference on Advances in Computer Entertainment Technology, Lissabon.

[13] Cuccurullo, S., Francese, R., Risi, M., \& Tortora, G. (2011). A visual approach supporting the development of microapps on mobile phones. 17. International Conference on Distributed Multimedia Systems, Florenz.

[14] AppBrain (2019). Android app frameworks. https://www. appbrain.com/stats/libraries/tag/app-framework/android-appframeworks?sort=apps. Zugegriffen: 10. Apr. 2020. 